\title{
Dall'analisi fenomenologica alla verifica sperimentale in psicologia: indagini sui meccanismi di difesa psichica in ambito educativo
}

\author{
Valeria Biasi
}

Università degli Studi «Roma Tre», Dipartimento di Scienze della Formazione

doi: 10.7358/ecps-2014-009-bias

valeria.biasci@uniroma3.it

FROM PHENOMENOGICAL ANALYSIS

TO EXPERIMENTAL VERIFICATION IN PSYCHOLOGY:

STUDIES ON PSYCHOLOGICAL DEFENCE MECHANISMS

IN THE EDUCATIONAL FIELD

\section{Abstract}

The present contribution examines the shift from introspective analysis, based on the phenomenological method, to the evaluation of introspective data collected by means of empirical methods, which go to define so-called "experimental Phenomenology", all within a perspective of the integration between experimental phenomenology and psychodynamic assumptions. This examination is conducted here with regard to psychological defence mechanisms and delineates the opposing processes of "perceptual defence» and "emphasizing" (facilitation, accentuation). After describing the main results achieved in the perceptological domain, where it is possible to adopt an orthodox experimental protocol, the attention shifts to field studies in the educational field regarding the teacher-student relation, according to an empirical methodology enabling a "quantification" of the presence of specific defence mechanisms which can arise in the educational relation, such as the overestimation or underestimation of phenomenal qualities attributed to oneself andlor to the other. Besides confirming the main dynamics of accentuation (overestimation) and defence (underestimation) demonstrated experimentally in laboratory studies, the contribution provides an example of the quantification of an essentially qualitative concept as the "defence mechanism", as is clear from its very definition: a defence mechanism is any behavioral strategy or attitude aimed at reducing andlor preventing subjectively perceived pain and suffering. This is a good opportunity, from a methodological 
standpoint, to demonstrate how it is possible to quantify strongly subjective variables in order to obtain an empirical verification, which is essential for arriving at scientifically credited knowledge.

Keywords: Educational relation, Experimental phenomenology, Perception, Psychodynamics, Psychological defence mechanism.

\section{INTRODUZIONE}

Il presente contributo esamina, nell'ottica di una integrazione tra fenomenologia sperimentale e assunti psicodinamici, il passaggio da un'analisi introspettiva basata sull'applicazione del metodo fenomenologico, alla valutazione dei dati introspettivi raccolti attraverso il ricorso a metodi empirici che definiscono il terreno della cosiddetta Fenomenologia sperimentale.

Questa duplice analisi viene condotta in quest'occasione nell'area dei meccanismi di difesa psichica ove vengono delineati i processi contrapposti della «difesa percettiva» e della enfatizzazione (facilitazione, risalto).

Vengono sintetizzate le relazioni, già esplorate e confemate in questo ambito nella letteratura di riferimento (Bonaiuto, 1965; Bonaiuto, Giannini, Biasi, Miceu Romano, \& Bonaiuto, 1997; Biasi, Calcagni, \& Parsi, 2008), fra condizioni osservative univoche o ambigue, ipotesi percettive covalenti o controvalenti, e conseguenti esiti osservabili di difesa o risalto, riepilogando, completando e superando, in alcuni casi, le conoscenze raggiunte grazie ai primi esperimenti funzionalisti.

L'attuale riscoperta dell'importanza delle influenze motivazionali nella percezione ha portato a nuove indagini, permettendo di verificare in modo sistematico l'intervento dei meccanismi di difesa psichica in relazione anche alle condizioni di contesto (o condizioni di osservazione). Fra questi esperimenti di «ultima generazione» si porta l'esempio - tra i vari possibili - dei processi di difesa percettiva verso incongruenze architettoniche, potenziate dal sovrappporsi di colorazioni «allarmanti e seriose», come il viola o il verde oliva, a confronto con colorazioni «rassicuranti e giocose», come il rosa e l'arancio. Come situazioni di controllo vengono presentate combinazioni di tali colorazioni con strutture visive non incongruenti.

Dopo la descrizione dei principali risultati conseguiti in ambito percettologico (riguardanti in particolare i meccanismi di difesa percettiva), ove è possibile permettersi il lusso di un protocollo sperimentale ortodosso, l'analisi viene spostata su indagini condotte in ambito educativo per quanto 
concerne la relazione docente-allievo, secondo una metodologia empirica che permette di «quantificare» la presenza di specifici meccanismi di difesa che possono intervenire nella relazione educativa, quali la sovrastima o la sottostima delle qualità fenimeniche attribuite a sé e/o all'altro.

Oltre ad una conferma delle principali dinamiche di enfatizzazione (sovrastima) e difesa (sottostima) già ampiamente dimostrate in sede sperimentale atttraverso le citate indagini di laboratorio, si vuole offrire un esempio di quantificazione di un dato sostanzialmente qualitativo quale quello rappresentato dal concetto meccanismo di difesa, così come appare già nella sua definizione: ossia, si definisce meccanismo di difesa qualunque strategia comportamentale o atteggiamento atti a ridurre elo prevenire il dolore e la sofferenza soggettivamente percepita. Si offre quindi l'occasione per dare dimostrazione di come, in qualità di ricercatori, possiamo riuscire a quantificare variabili a forte valenza soggettiva, al fine di ottenere una verifica empirica, momento indispensabile per il raggiungimento di conoscenze accreditate come scientifiche.

\section{POSIZIONI CLASSICHE E SVILUPPI INNOVATIVI IN TEMA DI DIFESE E RISONANZE NELLA PERCEZIONE}

Il reperire nessi fra ambiti di indagine diversi come la fenomenologia e la psicodinamica è volto ad incrementare i poteri esplicativi e predittivi attraverso una maggiore generalizzabilità dei ritrovati scientifici, anche con riferimento alla percezione umana.

Un'area cruciale da esplorare a tale proposito è quella dei processi e fenomeni della cosiddetta "difesa percettiva», o, all'opposto, della "risonanza» («enfatizzazione», «risalto», «facilitazione»).

L'approccio psicodinamico ai fenomeni cognitivi venne proposto già nella psicoanalisi classica (Freud, 1900) e venne di fatto applicato dal cosiddetto Funzionalismo, secondo Bruner (1992) e altri studiosi, negli anni quaranta, attraverso il cosiddetto New look on perception: un orientamento teorico che tendeva a dare rilievo alle influenze delle motivazioni o esigenze del percipiente su quanto percepito.

Sebbene i moteplici esperimenti condotti dai funzionalisti fornissero continue conferme dell'intervento delle variabili affettive e personologiche nell'elaborazione percettiva, restava il punto irrisolto costituito dal fatto che, come è esperienza comune, non basta desiderare per ottenere, né volere per percepire. Quindi occorreva spiegare quando, in quali condizioni di contesto, la proiezione dei desideri e la relativa conferma delle aspettative dell'osservatore si verificassero, e quando ciò, invece, non fosse possibile. 
Sulla base degli studi finora svolti è stato elaborato un quadro sinottico delle relazioni fra condizioni sperimentali ed effetti ottenibili nel quadro delle possibili elaborazioni percettive di immagini congrue (covalenti) o incongrue (controvalenti), esposto molto sinteticamente nella Tabella 1 (Bonaiuto, Biasi, \& Giannini, 2002; Biasi, 2006). Esaminando questa tabella è possibile cogliere il ruolo determinante delle "condizioni di osservazione». Lo schema segnala appunto le interazioni fra condizioni osservative e conseguenti modalità di elaborazione delle immagini, interessate $\mathrm{da}$ ipotesi percettive covalenti (immagini congruenti, armoniche e plausibili), oppure controvalenti (immagini incongruenti, conflittuali, paradossali). In sintesi, se le condizioni osservative sono univoche, come accade nella visione centrale, chiara e distinta, e l'immagine proposta per elaborare il percetto incontra in maniera consonante gli schemi mentali di riferimento (cosiddetta «ipotesi percettiva covalente»), allora si ottiene un effetto di risalto (enfatizzazione o magnificazione).

A tale proposito si vedano le dimostrazioni negli esperimenti classici di Bruner \& Goodman, 1947; nonché di Bruner \& Postman, 1949b; allorché aveva luogo la valutazione delle dimensioni di simboli positivi, che venivano ingranditi, in funzione del loro valore e interesse: un caso di risalto, per l'appunto. A parità di condizioni univoche di osservazione, se l'immagine da cui si parte per elaborare il percetto contraddice gli schemi mentali di riferimento («ipotesi percettiva controvalente»), nuovamente si ottiene di nuovo un effetto di risalto (enfatizzazione o magnificazione).

Si vedano in proposito gli esperimenti classici di Bruner e Postman (1949b) sulla valutazione delle dimensioni di simboli sgradevoli (come la svastica): acquista rilievo anche un'immagine conflittuale e sgradevole, in qualche modo saliente e importante, da segnalare. Questi risultati contrastavano rispetto all'ipotesi iniziale degli sperimentatori secondo i quali ci si doveva aspettare in generale una difesa dalla percezione di tali contenuti allarmanti.

Tabella 1. - Schema riassuntivo delle condizioni osservative e delle conseguenti modalità di elaborazione delle immagini interessate da ipotesi percettive covalenti (immagini congruenti, armoniche e plausibili) oppure controvalenti (immagini incongruenti, conflittuali e paradossali).

CONDiZIONi D’OSSERVAZIONE

\begin{tabular}{|c|c|c|}
\hline & Centrali (univoche) & Marginali (ambigue) \\
\hline & \multicolumn{2}{|c|}{ Effetti ottenibili } \\
\hline Covalenti & Risalto & Facilitazione \\
\hline Controvalenti & Risalto & Difesa \\
\hline
\end{tabular}


Diverso è il caso delle condizioni di osservazione ambigue, vale a dire della percezione "marginale» (a tempi molto brevi, o semi-mascherata, o comunque difficoltata). In tal caso, l'ipotesi percettiva covalente dà nuovamente luogo ad effetti di facilitazione, ossia nuovamente di risalto, di evidenziazione; come è accaduto negli esperimenti di Levine, Chein \& Murphy, 1942; Schafer \& Murphy, 1943; Postman, Bruner, \& McGinnies, 1948; McClelland \& Atkinson, 1949; Vanderplas \& Blake, 1949; Wispè \& Dranbarean, 1953. Queste indagini hanno mostrato vari tipi di salienza: per vividezza di qualità funzionali, per emergenza di figura sullo sfondo, per magnificazione di simboli, per abbassamento delle soglie di riconoscimento (visive o uditive), ecc.

$\mathrm{Al}$ contrario, l'ipotesi percettiva "controvalente», ossia la contraddizione rispetto alle aspettative, favorisce, per l'appunto nella sola condizione di ambiguità, la difesa percettiva: come registrato anche nell'esperimento classico di Bruner e Postman (1949a) sulle colorazioni incongrue di carte da gioco presentate al tachistoscopio che per essere riconosciute richiedevano tempi di esposizione maggiori (l'allungamento dei tempi di riconoscimento è indice di difesa percettiva). Nello specifico si ritiene quindi, e ciò in linea con gli originari studi funzionalisti, che il percetto sia elaborato dal percipiente in relazione alle sue aspettative e agli schemi mentali già consolidati, comprensivi anche dei bisogni dell'osservatore. Riassumendo, l'immagine percepita incontra lo schema mentale di riferimento, il quale funge da «ipotesi covalente» se è in armonia col percetto, dando origine ad una immagine congrua. Se l'immagine percepita contrasta invece con lo schema mentale già acquisito, trovandosi cioè in rapporto di opposizione, si produce il vissuto di un'immagine incongrua, paradossale, bizzarra.

In condizioni osservative univoche, cioè in visione chiara e distinta, l'immagine congrua viene elaborata, acquisendo risalto, divenendo saliente per il percipiente. In condizioni osservative ambigue l'immagine congrua dà luogo a facilitazione percettiva, con una sostanziale accettazione, vale a dire con buona coesione tra percetto e desiderio.

Con immagini viste attraverso vetri smerigliati (Levine, Chein, \& Murphy, 1942), i soggetti tenuti a digiuno per varie ore tendevano ad attribuire significati di cibi a forme incerte, semi-mascherate, di oggetti posti dietro a quei vetri. Si trattava di fenomeni di facilitazione percettiva dovuti al processo di assimilazione tra percetto ed ipotesi percettiva. Sempre in condizioni ambigue venivano mostrati da Postman, Bruner e McGinnies (1948) dei simboli verbali collegati ad interessi religiosi, economici, politici: col risultato che i percipienti riconoscevano prima, cioè a soglie di riconoscimento inferiori, $\mathrm{i}$ simboli in accordo con i loro interessi.

In condizioni osservative ambigue, l'immagine incongrua può dare luogo a processi di accomodamento e relativa regolarizzazione. Al contrario, in con- 
dizioni osservative univoche, l'immagine incongrua viene elaborata attraverso la segnalazione e l'allarme, comportando effetti di risalto.

Ciò permette oggi di spiegare i risultati sperimentali poco comprensibili con le classiche teorie funzionaliste; e cioè per esempio quella parte di dati raccolti da Bruner e Postman (1947), nel caso dell'esperimento sulla stima di dischi con decorazioni allarmanti quali il simbolo della svastica, prima descritti. In sintesi, in visione libera, chiara e distinta, cioè univoca, gli autori registrarono risultati in cui dischi con le svastiche venivano magnificati (cioè leggermente sovrastimati nella loro grandezza), così come altri dischi decorati con il simboli del dollaro, nonostante la valenza emotiva contrapposta. Non scattava cioè il processo della difesa percettiva, che gli sperimentatori si attendevano, ma che era un'operazione non possibile in visione chiara e distinta, se non attraverso un forte distacco dalla realtà, come nelle allucinazioni.

In altri termini, l'effetto di risalto da ipotesi controvalente, con produzione quindi di immagini incongrue, dissonanti, allarmanti, è stato verificato nelle condizioni osservative univoche riguardanti la stima del cerchio con il simbolo della svastica, che gli autori funzionalisti con il loro sistema di riferimento teorico non erano ancora pronti a spiegare. Essi poterono registrare fenomeni di difesa percettiva soltanto con immagini sgradite, in condizioni osservative ambigue.

Le condizioni univoche di osservazione corrisponderebbero ad una preminenza del "principio di realtà», mentre nelle condizioni ambigue vi sarebbe spazio per i fenomeni di "proiezione» e di gratificazione pulsionale. Negli stati di ridotta vigilanza e in quelli alterati di coscienza (stati psicotici, uso di droghe, ecc.) troviamo un estendersi della condizione di ambiguità, con le relative conseguenze.

Come hanno ricordato recentemente Balcetis e Dunning (2006), gli esperimenti riferiti dai funzionalisti subirono fin dall'inizio obiezioni metodologiche, tendenti a sminuire il peso delle componenti motivazionali focalizzate nelle ipotesi di lavoro. Ad esempio la sopravalutazione della grandezza delle monete da parte dei «bambini poveri» di Bruner e Goodman poteva essere connessa alla mancanza di familiarità col denaro, o a problemi di personalità $\mathrm{o}$ all'intervento della memoria piuttosto che della percezione. Inoltre certi rallentamenti nel riconoscimento di immagini o parole inquietanti potevano significare l'interferenza della sorpresa o dell'imbarazzo di fronte a quei contenuti problematici. Tuttavia conferme dell'esistenza di tali reazioni difensive vennero dalle indagini centrate sulla registrazione del riflesso psicogalvanico (con aumento della sudorazione del palmo della mano) in corrispondenza di una percezione subliminale (cioè sotto il livello di coscienza) di immagini allarmanti (come appunto delle parole tabù). Vi fu anche il caso di immagini pubblicitarie inserite grazie ad un solo fotogramma nell'ambito di 
un filmato, e che quindi vengono percepite sempre sotto il livello di coscienza in quanto l'osservatore non riconosce un oggetto a tale velocità di proiezione (cosiddetta "pubblicità subliminale», oggi in genere vietata), situazione che produsse un aumento della richiesta del prodotto in questione - una nota bibita americana - nell'audience ignaro.

Attualmente, esaminando la letteratura teorico-sperimentale più recente, si assiste ad una riscoperta dell'importanza delle influenze motivazionali nella percezione e nei processi cognitivi in genere. Si fa spesso ricorso a influenze top-down oppure alla Signal Detection Theory Analysis (Theios \& Jenison, 1999; Holyoak \& Morrison, 2005). In particolare due autori, Molden e Higgins (2005) parlano d'una «seconda generazione di ricerche sull'interfaccia motivazione/cognizione».

In Italia, già alla fine degli anni ' 50 Canestrari (1955 e 1957) e Canestrari e Marzi (1958) avevano svolto opera di utile divulgazione in merito alla conferma sperimentale di assunti psicodinamici tipici, con una posizione di relativa continuità rispetto alla tradizione psicoanalitica.

Un altro contributo italiano in linea con l'approccio funzionalista è stato quello della spiegazione degli effetti percettivi ottenibili sperimentalmente con la monotonia, vale a dire a seguito della «privazione sensoriale» e della "fissazione prolungata». Interpretando la monotonia come «saturazione d'omogeneità", il viraggio dello stile percettivo in direzione analitica venne predetto e interpretato come attivazione motivazionale verso l'eterogeneità delle strutture (Bonaiuto, 1965, 1970, 1984).

Più recentemente autori come Weinberger, Siegel e Decamello (2000), hanno auspicato integrazioni metodologiche allorché i metodi di ricerca delle scienze cognitive vengano applicati per verificare costrutti psicoanalitici, come la dinamica motivazionale ed altri fondamenti tipici, al fine di arricchire il modello della mente elaborato dalle scienze cognitive.

In realtà vi sono stati in passato periodi, come i decenni ' 60 , '70 e '80 del secolo scorso, di forte resistenza e diremmo proprio di oscurantismo per quanto riguarda l'accettazione di queste ed altre integrazioni. Il numero di lavori centrato sulla dinamica dei processi affettivi si ridusse enormemente negli anni dell'affermarsi del primo cognitivismo nelle sue branche più estreme.

Vi furono però in quegli anni alcuni rari ma preziosi tentativi di collegamento tra opposte tendenze scientifiche. Ricordiamo a questo proposito il lavoro pubblicato nel 1974 da Erderly con il titolo $A$ new look at the new look: Perceptual defense and vigilance, dove si definisce il fenomeno della difesa come un effetto di vigilanza, uno speciale effetto di selettività nel cognitive processing. Tale selettività, compreso il costrutto di selettività difensiva, entrerebbe in gioco in modo pervasivo nel continuum dell'elaborazione delle informazioni (dal momento dell'input a quello dell'output). 
Come è noto un paradigma scientifico affonda comunque le sue radici nella realtà storica, come ci possono mostrare gli studi di storia della scienza, tuttavia non mancheremo mai di sottolineare quanto dannosi possono risultare, per il cammino dei saperi, quei lunghi periodi di resistenza persi a combattere le mode culturali e scientifiche. Occorre aspettare momenti di revisione e di ricognizione più equilibrati, cioè lontani dai cosiddetti «fondamentalismi» che appunto inquinano ogni tanto anche il pensiero scientifico, provocandone momentanei arresti.

In sostanza oggi ci sembra che gli studi di fenomenologia sperimentale, con gli sviluppi successivi, offrano l'occasione per una migliore integrazione fra lo studio dei processi cognitivi e quello dei processi affettivi anche nell'ambito delle varie applicazioni possibili, tra cui quelle rivolte al settore educativo.

In riferimento alle condizioni osservative ambigue sono stati riferiti poc'anzi alcuni fra i principali esperimenti funzionalisti per la difesa percetti$v a$, mentre in riferimento alle condizioni osservative univoche riporteremo di seguito alcuni recenti studi sui fenomeni di enfatizzazione percettiva.

Dagli anni '80 del '900 ai giorni nostri il risalto delle immagini incongrue nelle condizioni osservative univoche è stato più volte verificato in vari esperimenti nel Laboratorio diretto da Bonaiuto in Roma, dalla cui analisi si conferma che nelle condizioni di ambiguità percettiva vengono registrati fenomeni di attenuazione e mascheramento delle incongruenze; si attiva cioè un processo di assimilazione pro-attiva dell'immagine percettiva rispetto agli schemi mentali preesistenti, di cui è portatore il percipiente.

Nella condizione di univocità percettiva, invece, essendo la visone chiara e distinta, non è più possibile difendersi dall'incongruità e dal conflitto attraverso mascheramenti e dinieghi, dovendo tener conto degli indicatori di realtà. Si verificano allora dei processi di enfatizzazione e risalto delle incongruenze, favoriti dal contrasto pro-attivo rispetto agli schemi mentali di cui è portatore il percipiente. Tale interpretazione, elaborata dal nostro gruppo di ricerca e ormai collaudata sperimentalmente, appare sostenuta da molteplici evidenze empiriche, di cui daremo una breve sintesi nel paragrafo che segue.

\section{ESPERIMENTI DI ULTIMA GENERAZIONE SULLE DIFESE PERCETTIVE}

Una varietà importante di incongruità percettiva, sottoposta a processi di elaborazione, è quella che si può cogliere nelle raffigurazioni di oggetti aventi posizioni, oppure forme, oppure colorazioni contraddittorie rispetto alla co- 
mune esperienza. In questi casi Bonaiuto, Giannini e Bonaiuto (1990) poterono accertare l'importanza fondamentale delle condizioni di osservazione.

Nelle condizioni di ambiguità l'osservatore appare preferire la conferma degli schemi e una relativa difesa dalle irregolarità, il secondo tipo di condizioni osservative - cosiddette "univoche» - mette in luce la possibilità di dinamiche opposte, non meno importanti per le spiegazioni e previsioni che possono venire avanzate in questo campo.

Le situazioni sperimentali studiate in Roma comprendono, come si accennava, anomalie architettoniche riprodotte con illustrazioni e con modelli tridimensionali come edifici pendenti, o edifici fratturati, o edifici con facciate in disordine, e, in più casi, presentati all'osservatore secondo vari orientamenti, favorevoli di volta in volta all'ambiguità o all'univocità percettive.

La prima indagine veniva comunque impostata su un edificio pendente reale, la casa a due piani costruita nel Rinascimento con una certa inclinazione, nel Sacro Bosco di Bomarzo, il parco della Villa Orsini. Un gruppo di allievi fu invitato a valutare sul campo, con un semplice goniometro, l'inclinazione apparente dell'edificio, standovi dentro. Si notò una consistente sovrastima dell'inclinazione rispetto alla verticale: valutata in media 22 gradi anziché 13 gradi. La prova fu ripetuta con un'illustrazione schematica della Casa di Bomarzo, tale da formare con la linea di base un angolo di 15 gradi. Vennero inoltre fatte valutare altre due sagome di controllo, di uguale forma e inclinazione, l'una consistente di un semplice rettangolo, l'altra corredata da linee per ottenere una maggiore complessità. L'indagine fu svolta con 50 partecipanti adulti, d'ambo i generi, esaminati individualmente e con rotazione delle prove. La sagoma con caratteri di edificio dette luogo ad una sovrastimazione media della posizione anomala (16 gradi e mezzo anziché 15 gradi), la quale non compariva con le due sagome di controllo, prive dell'aspetto di edificio (Bonaiuto, Giannini, \& Bonaiuto, 1990).

Per una successiva indagine, più complessa, furono allestiti dei modelli tridimensionali, ben dettagliati, di edifici noti: una "Torre di Pisa» (pendente di 7 gradi rispetto alla verticale) e un "Campanile di S. Marco» (con la stessa inclinazione). Furono allestiti inoltre due elementi di confronto, detti "Non-edifici», che avevano le stesse forme, proporzioni e inclinazioni dei modelli anzidetti, ed anche una decorazione casuale delle pareti che li rendeva complessi, ma senza l'aspetto tipico degli edifici. Questi quattro modelli vennero presentati uno alla volta, ciascuno secondo dodici orientamenti diversi rispetto all'osservatore. I partecipanti, esaminati individualmente, dovevano valutare la pendenza apparente, ogni volta, per confronto con una scala di comparazione che recava sette possibili inclinazioni: una esatta, tre superiori e tre inferiori rispetto ai 7 gradi dei modelli: secondo il «metodo dei limiti». 
Nei risultati apparve chiaro che le condizioni di osservazione ambigue, quando cioè il modello architettonico appariva pendente verso l'osservatore o verso l'opposto, davano luogo a sottoestimazione della pendenza, mentre le condizioni di osservazione univoca, cioè con pendenza chiaramente visibile verso destra o verso sinistra, davano luogo a nette enfatizzazioni dell'inclinazione anomala. Tale enfatizzazione risultò più cospicua (fino ad oltre 10 gradi) con il modello incongruo del Campanile di S. Marco, il quale corrispondeva ad uno schema mentale rigorosamente verticale; mentre per la Torre di Pisa lo schema mentale correlato è appunto quello di una Torre pendente: diper sé incongrua rispetto allo schema generale di edificio, ma in qualche modo relativamente familiare e più accettabile. I "Non-edifici», per i quali non esistevano schemi mentali espressivi di verticalità, non davano luogo all'enfatizzazione (cfr. anche Biasi, Chiappetta Cajola, \& Bonaiuto, 2010, per la conferma di simili osservazioni con soggetti in età dello sviluppo).

Successivamente gli stessi autori hanno provato ad influire su queste dinamiche manipolando, ad esempio, l'importanza dello schema mentale, facendo precedere opportune descrizioni verbali; oppure manipolando gli atteggiamenti individuali di intolleranza o tolleranza dell'incongruità; e ciò sia con istruzioni verbali, sia con «messaggi» non verbali, cioè con caratteristiche cromatiche e simboli opportunamente assegnati ai modelli presentati.

Differenze significative si sono quindi verificate allorché, ad esempio, al conflitto strutturale tipico dell'incongruenza architettonica si è aggiunta un'ulteriore atmosfera conflittuale, data dall'impiego di colorazioni diffuse di tipo «allarmante» (tonalità grigio-violacea o verde oliva); mentre effetti opposti si sono ottenuti con i colori «rassicuranti» (tonalità rosa acceso e arancio).

Fra le incongruenze architettoniche sono state focalizzate quelle di sagome di edifici pendenti o di edifici a «baionetta», con risultati concordanti.

Nei nuovi esperimenti la stessa sagoma d'un edificio a torre inclinato di 7 gradi rispetto alla verticale è stata stampata su un cartoncino formato A4 uniformemente colorato con tinte «allarmanti», quali il viola o il verde oliva, oppure con tinte «rasserenanti», quali il rosa acceso e l'arancio.

Sono stati anche svolti confronti con l'impiego di colorazioni emotivamente intermedie, quali la tinta color sabbia. Su queste indagini hanno riferito Bonaiuto, Biasi, Giannini, Calcagni, \& Bellini, 2007; Biasi, Calcagni, \& Parsi, 2008; Bonaiuto, Biasi, Giannini, Calcagni, \& Parsi, 2008.

La valutazione del grado di anomalia architettonica, cioè della pendenza, veniva svolta individualmente da ciascun partecipante utilizzando una scala di comparazione a sette passi. I risultati hanno chiarito che nel caso delle tonalità allarmanti compaiono manifestazioni difensive verso la struttura conflittuale, nel senso che la pendenza anomala viene sottostimata o comunque non raggiunge valori medi particolarmente intensi. Con le tona- 
lità «rassicuranti» compaiono vistosi effetti di enfatizzazione dell'anomalia architettonica e cioè una significativa sovraestimazione della pendenza. Con la tonalità intermedia si sono ottenute valutazioni disposte a metà strada fra le precedenti.

Altri confronti, di notevole importanza metodologica, sono stati effettuati con l'impiego di strutture non conflittuali. Queste ultime presentano peculiarità di posizione analoghe a quelle delle sagome di edifici pendenti, ma, a differenza di queste non contraddicono schemi mentali consolidati nell'osservatore. Si è trattato, in particolare, dell'immagine di una gru vista di profilo; e di quella di una barra di passaggio a livello. Entrambe queste strutture si presentano inclinate di sette gradi rispetto alla verticale, come nel caso delle sagome di edifici. Si tratta però di oggetti per i quali è normale e plausibile qualsivoglia inclinazione, entro certi limiti. Sono state impiegate anche in questo caso opportune scale di comparazione a sette caselle, per un adeguato matching, caso per caso. Con queste sagome, le aggiunte delle stesse colorazioni diffuse impiegate per gli edifici, per quanto contrapposte, non hanno dato luogo né a difese né ad enfatizzazioni.

La seconda incongruenza architettonica posta allo studio è data dalla sagoma d'un edificio cosiddetto "a baionetta», ossia caratterizzato da una linea di frattura a metà del corpo, con uno slittamento del $27 \%$ della parte superiore rispetto all'inferiore. Anche questa sagoma, a contorno lineare nero sottile, è stata stampata su cartoncini A4 con le stesse tinte adoperate nell'esperimento precedente. In questo caso ciascun partecipante, sempre esaminato individualmente, aveva a disposizione una specifica scala di comparazione a sette passi. I risultati sono analoghi a quelli precedenti: anche per quanto riguarda l'esito di una situazione di controllo costituita dalla sagoma di una «libreria», caratterizzata da un identico livellamento della parte superiore rispetto all'inferiore. L'aggiunta delle stesse colorazioni diffuse impiegate per l'edificio «a baionetta» non ha dato luogo né a difese né ad enfatizzazioni, costituendo quindi una riprova sperimentale interessante.

In sostanza questi esperimenti dimostrano il ruolo giocato, nell'elaborazione percettiva, dagli schemi mentali dell'osservatore e dalle conseguenti aspettative, dalla capacità di leggere le qualità emotive veicolate dal colore in termini di livello di conflittualità percepito, e di tenere in considerazione le condizioni di osservazione (secondo il principio di realtà). Cognizione ed affettività vengono visti in stretta interdipendenza, secondo la lettura psicodinamica classica, e si offre una continua rilevazione empirica degli effetti prodotti da questo rapporto dinamico. 


\section{DALL'ANALISI FENOMENOLOGICA CONDOTTA SUI MECCANISMI DI DIFESA ALLA VERIFICA EMPIRICA: APPLICAZIONI IN AMBITO EDUCATIVO}

Com'è noto, la ricerca psicologica ha consentito la progressiva individuazione di vari meccanismi di difesa, intesi ad evitare o ridurre stati di fastidio, sofferenza, dolore; a loro volta dipendenti da conflitti psichici e condizioni di stress di intensità superiore alle soglie individuali di tolleranza. Queste dinamiche si presentano in ambito clinico come in altri contesti, caratterizzando, senza soluzioni di continuità, sia la normalità sia la patologia.

Una lista aggiornata di oltre trenta differenti meccanismi difensivi (Tabella 2), con una descrizione dei processi relativi e con vari riferimenti anche sperimentali, nonché una elencazione di ulteriori forme d'illusione emotivamente rilevanti, sono state compilate da Bartoli e Bonaiuto (1997), anche sulla scorta di definizioni ed elenchi reperibili in molteplici trattazioni: sia classiche (da Sigmund Freud, ad Anna Freud, a Melanie Klein), sia recenti (Perry \& Cooper, 1987; Vaillant, 1992; Lingiardi \& Madeddu, 1994; Cramer, 2000; Parker \& Wood, 2008; cfr. inoltre il DSM-V dell'American Psychiatric Association, 2013, trad. it. Cortina, 2014).

Tabella 2. - Elencazione dei principali meccanismi di difesa psichica

(in ordine alfabetico; Bartoli \& Bonaiuto, 1997).

\begin{tabular}{cc}
\hline Acting out & Intellettualizzazione \\
Affiliazione & Ipocondria \\
Aggressione & Isolamento \\
Aggressione passiva & Negazione \\
Altruismo & Proiezione \\
Annullamento retroattivo & Razionalizzazione \\
Ascetismo & Regressione \\
Controllo onnipotente & Repressione \\
Conversione somatica & Rimozione \\
Diniego & Riparazione \\
Dissociazione & Scissione \\
Fantasticheria & Spostamento \\
Formazione reattiva & Stereotipia \\
Idealizzazione & Sublimazione \\
Identificazione & Svalutazione \\
Identificazione proiettiva & Umorismo \\
Imitazione & $\ldots$ \\
\hline
\end{tabular}


Molto vario si presenta il panorama dei metodi di valutazione, legati sia ai criteri adottati per la descrizione e classificazione dei meccanismi difensivi, sia alle procedure di individuazione: attraverso l'esame di contenuti e modalità espressive nei colloqui, di altri comportamenti pratici osservati direttamente o dopo registrazione, di risposte orali o scritte a test proiettivi, di risposte scritte a domande standardizzate, di punteggi assegnati con scale di valutazione applicate dal soggetto stesso (self-reports) o da esaminatori esterni.

Una tecnica consolidata (Biasi, Bartoli, \& Squarcia, 2000) si basa sull'analisi delle narrazioni autobiografiche rese, in questo caso, da insegnanti, con riferimento sia a contenuti descrittivi del sé, sia ad aspetti linguistici non casuali (scelte lessicali, sintattiche, grafiche, ecc.), sia ad eventuali aspetti di corrispondenza o discrepanza fra il contenuto manifesto e quello latente. La procedura, che va affidata ad esaminatori sufficientemente sensibili e preparati sotto il profilo psicodinamico, risulta analoga rispetto alle tecniche classiche di valutazione del colloquio: anche perché si tratta pur sempre di materiale verbale (senza integrazioni o interferenze dovute ad altri aspetti o canali espressivi). D'altra parte la narrazione scritta offre ulteriori peculiarità e possibilità, fra cui quella di analisi effettuabili in assenza sia di altre forme di registrazione, sia della persona stessa. Consente ancora di superare distanze di tempo e di spazio o determinate forme di chiusura e di inibizione comunicativa.

Rimanendo nell'ambito del contesto applicativo di tipo educativo-formativo, vediamo adesso come rappresentazioni (schemi) mentali e atmosfere emotive incidono sulla percezione di sé e dell'altro (quindi sulla strutturazione del mondo fenomenico inteso sia come realtà interiore sia come realtà percepita come incontrata) nel rapporto tra insegnanti e alunni adolescenti.

\subsection{Quadro teorico e ipotesi dell'indagine}

La ricerca condotta da Biasi, Bartoli e Amann Gainotti (2004) che qui viene riassunta tiene conto di studi precedenti sulle dinamiche motivazionali e sulle caratteristiche cognitive dell'adolescenza rispetto alla fanciullezza e alla preadolescenza (Bonaiuto \& Bartoli, 1967, 1970; Canestrari, 1970; Lenhart \& Rabiner, 1995; Dolto, 1996; Pietropolli Charmet, 2000; Palmonari, 2001; Amann Gainotti, 2002; Disanto, 2002; Fonzi, 2002).

Sono state prese in esame le rappresentazioni (schemi, ipotesi precostituite, aspettative) che gli adulti, in particolare gli insegnanti, sviluppano sul tema degli adolescenti e delle loro problematiche. Tali rappresentazioni risultano di particolare rilievo in quanto possono tendere ad orientare in maniera diversificata le pratiche educative: in termini di controllo o di guida, 
di tolleranza o intolleranza, rispetto ai comportamenti adolescenziali tipici. L'obiettivo principale è stato quello di indagare eventuali relazioni tra autovalutazioni del docente circa proprie caratteristiche personali, e immagini della condotta e degli atteggiamenti del discente (per esempio: valutare se stessi come efficienti, seri, rispettosi delle regole sociali e, in relazione a ciò, conferire un risalto maggiore o minore alle tendenze trasgressive e regressive tipiche della fase adolescenziale, ecc.).

Sul piano clinico, oltre che dinamico, lo sviluppo di queste conoscenze appare proficuo ai fini della comprensione, previsione e prevenzione di situazioni di sofferenza e di rischio, nell'ottica della promozione di relazioni interpersonali meno conflittuali e più costruttive, tra adolescenti ed adulti.

\subsection{Metodo, strumenti, partecipanti}

È stato somministrato a insegnanti di scuole medie inferiori e superiori di Roma e provincia, un questionario, appositamente costruito, con 14 domande chiuse e aperte, comprendenti anche liste di aggettivi fra cui scegliere per valutare se stessi, da un lato, e l' "adolescente tipico", dall'altro, ciò allo scopo di evidenziare le rappresentazioni che ogni docente costruisce sia su di sé sia sull'adolescente.

Per quanto concerne le rappresentazioni sugli adolescenti, vengono prese in considerazione diverse componenti psicologiche, attinenti alle aree cognitiva, affettiva e socio-relazionale, in modo da pervenire ad una valutazione dettagliata di atteggiamenti e comportamenti tipici di questa fase evolutiva.

Hanno partecipato alla ricerca 140 docenti, di cui 106 donne e 34 uomini, differenziati per tipo di specializzazione didattica nelle discipline umanistiche $(35.8 \%)$ e scientifiche $(64.2 \%)$. L'età dei docenti partecipanti è compresa tra i 28 e i 65 anni (età media: 46 anni e 9 mesi).

\subsection{Modalità di elaborazione dei dati qualitativi attraverso l'applicazione di indici quantitativi e risultati conseguiti}

$\mathrm{Si}$ è ritenuto opportuno misurare le corrispondenze tra auto-valutazioni date dagli insegnanti e valutazioni dell' adolescente tipico date dagli stessi. Nel primo compito il docente doveva indicare almeno 10 aggettivi (scegliendoli da un'ampia lista di 48 voci comprendenti aggettivi a diversa tonalità emotiva descrittivi di possibili aspetti del sé).

Nel secondo compito lo stesso docente elaborava una risposta alla seguente domanda: «Come definirebbe, attraverso una serie di 10 aggettivi di 
sua scelta, gli adolescenti di oggi?». Qui di seguito illustriamo la procedura di quantificazione dei dati e i risultati ottenuti. Sono stati inizialmente categorizzati - con il metodo dei tre giudici indipendenti - i molti aggettivi adottati dagli insegnanti per descrivere se stessi e gli adolescenti, distinguendo aggettivi con polarità positiva e aggettivi con polarità negativa (Tabella 3). Si fa presente che sono stati individuati anche degli aggettivi ambigui, intermedi o non discriminativi, presenti in quantità ridotta e quindi non utilizzati nelle computazioni.

Si è proceduto poi al calcolo di due indici individuali per ogni insegnante, il primo pertinente all'immagine di sé, il secondo riguardante l'immagine dell'adolescente.

Ciascun indice è ottenuto, nell'ambito dell'area pertinente, dalla somma algebrica degli aggettivi positivi $(+1)$ e negativi $(-1)$ diviso per il numero di aggettivi forniti. Ciò, dunque, sia per le auto-valutazioni, sia per le valutazioni dell'adolescente. Gli indici così formati hanno presentato una gamma di variazioni inter-individuali $\mathrm{da}+1.00$ (massimo) a -0.40 (minimo), per le auto-descrizioni; e da +1.00 (massimo) a -1.00 (minimo) per la figura dell'adolescente.

Per quanto riguarda i rapporti tra questi due ordini di valutazioni, si riscontra una tendenza generale ben rappresentata nella Figura 1, che riassume i dati ottenuti da tutti i 140 insegnanti.

Tabella 3. - Esempi di aggettivi "positivi» e «negativi» espressi dagli insegnanti intervistati, con riferimento all'immagine di sé o a quella dell'adolescente tipico.

\begin{tabular}{cc}
\hline Aggettivi «Positivi» & Aggettivi «NEgativi» \\
\hline Calmo & Aggressivo \\
Creativo & Brusco \\
Curioso & Distratto \\
Ingegnoso & Impaziente \\
Intelligente & Impulsivo \\
Ottimista & Inquieto \\
Piacevole & Insoddisfatto \\
Pieno di risorse & Preoccupato \\
Responsabile & Sottomesso \\
Spontaneo & Superficiale \\
$\ldots$ & $\ldots$ \\
\hline
\end{tabular}




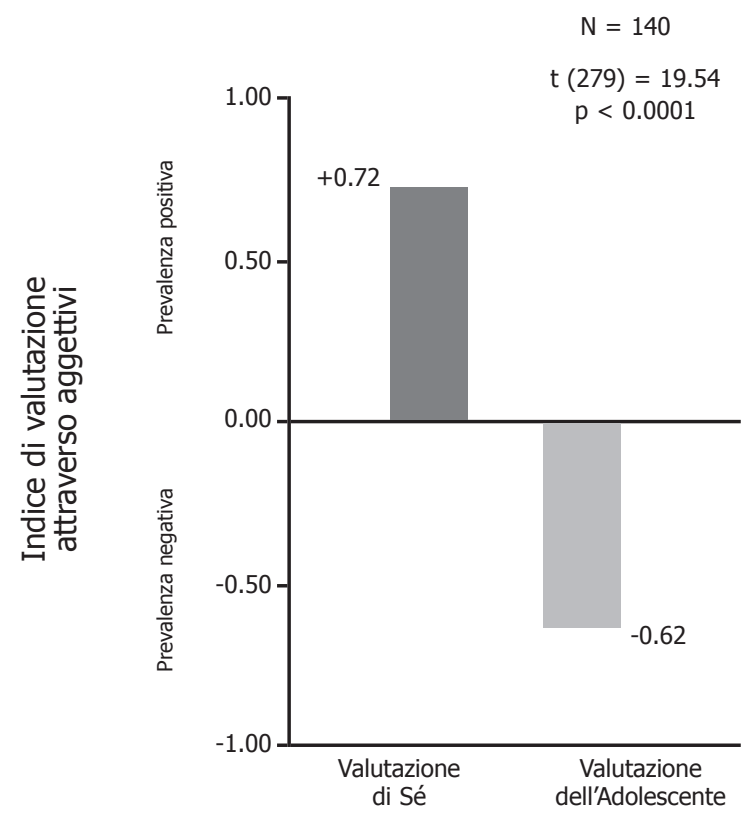

Figura 1.

Come si può osservare i docenti, in media, mostrano di fare frequente ricorso al rilievo di aspetti negativi della condotta del giovane, in contrapposizione al rilievo di aspetti positivi attribuiti a se stessi. Ciò può riflettere un certo realismo, vista la complessità della realtà psicologica adolescenziale, ma anche una possibile inclinazione all'uso di tecniche difensive quali la scissione di aspetti contraddittori del sé e la proiezione all'esterno di una parte di tali aspetti, con l'esito di spostare sulla relazione con l'adolescente nuclei conflittuali interni, strettamente personali, che proprio da tale non facile relazione possono essere riattivati. Una simile procedura difensiva può venire adottata da alcuni insegnanti anche per far fronte al carico di stress lavorativo; ricoprirebbe quindi un originario intento adattivo, al costo di una deformazione dell'immagine dell'altro.

Scendendo in maggiori dettagli, è molto interessante il comportamento di un sottogruppo ristretto di 15 insegnanti (10.7\% del totale; Figura 2) che seguono una tendenza divergente: appaiono meno categorici, più tolleranti e meno punitivi nel considerare gli atteggiamenti adolescenziali. 


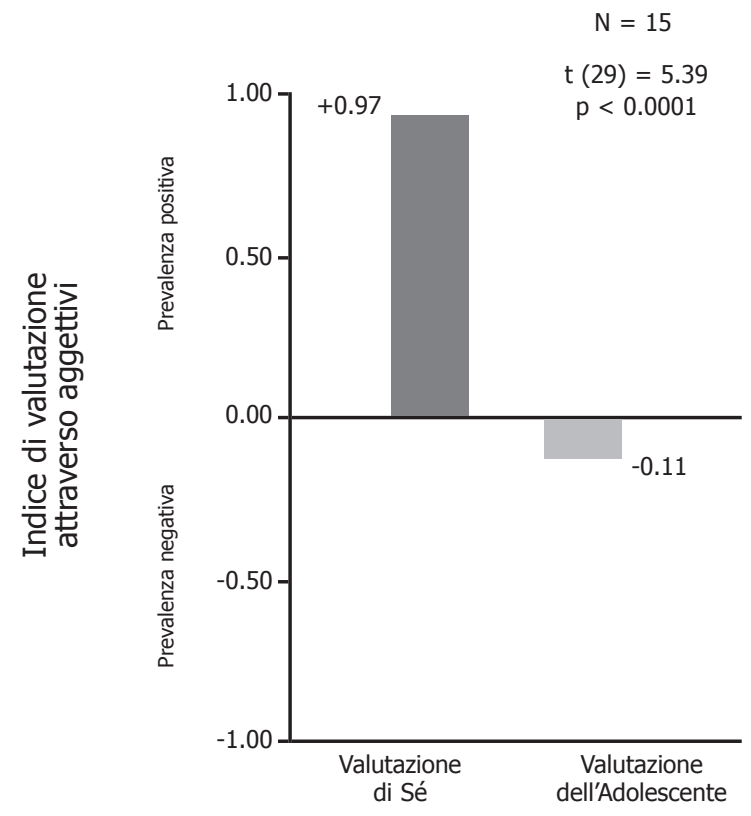

Figura 2. - Insegnanti che utilizzano il meccanismo difensivo della idealizzazione.

Si tratta di docenti che valutano se stessi in modo del tutto positivo e, con riferimento agli adolescenti, sottolineano prevalentemente gli aspetti costruttivi ed evolutivi che caratterizzano questa fascia d'età (vengono elencati come comportamenti che incontrano l'approvazione dell'adulto quelli espressivi di "spontaneità», "sincerità», "curiosità ed interesse», "spirito di gruppo»). Tali insegnanti, in altri termini, sembrano utilizzare in maniera globale, nella relazione il meccanismo della idealizzazione.

È stato possibile identificare un altro sottogruppo di insegnanti con comportamenti estremi, nel senso che danno valutazioni del tutto positive alla propria persona e completamente negative alla persona dell'adolescente. Si nota che questi insegnanti, anch'essi in numero di 15 (10.7\% del totale), rappresentano l'adolescente tipico del tutto negativamente, definendolo come «impaziente», "aggressivo», «inquieto", "distratto», «impulsivo»; e non indicano mai, come invece succede per il gruppo più ampio restante (pari a 110 insegnanti ossia il 78.6\%), aspetti positivi quali "pieno di iniziativa», "pieno di risorse», «entusiasmabile» e «spontaneo». 
Il medesimo sottogruppo segnala come decisamente fastidiose alcune caratteristiche proprie dell'adolescente, definite come "cattiva educazione», "superficialità», "mancanza di rispetto», «arroganza». Potrebbero essere attivi, in questo caso, elementi difensivi di tipo svalutante, correlabili ad ulteriori meccanismi di scissione-proiezione all'esterno del negativo e di razionalizzazione davanti a situazioni relazionali difficili (Figura 3).

Confrontando i punteggi ottenuti, in termini di indice individuale medio, dagli insegnanti che utilizzano l'idealizzazione del sé e dell'altro, oppure la idealizzazione del sé e la svalutazione dell'altro, si dimostra la significatività delle differenze sia per la variabile «valutazione di sé» ( $28=1.98 ; \mathrm{p}<0.03$ ), sia per la «valutazione dell'adolescente» (t $28=8.85 ; \mathrm{p}<0.001$ ). I sottogruppi menzionati, inoltre, differiscono significativamente anche dall'ampio gruppo dei restanti 110 intervistati (variabile "Valutazione di Sé»: per gli insegnanti che idealizzano rispetto ai 110 restanti: t $123=4.14$; $p<0.001$; per gli insegnanti che proiettano rispetto ai 110 restanti: t $123=3.02 ; \mathrm{p}<0.01$; variabile "Valutazione dell'Adolescente»: per gli insegnanti che idealizzano rispetto ai 110 restanti: t $123=4.14 ; \mathrm{p}<0.001$; per gli insegnanti che proiettano rispetto ai 110 restanti: t $123=3.53$; p < 0.001; cfr. Figura 4). Ciò conferma l'opportunità delle distinzioni operate.

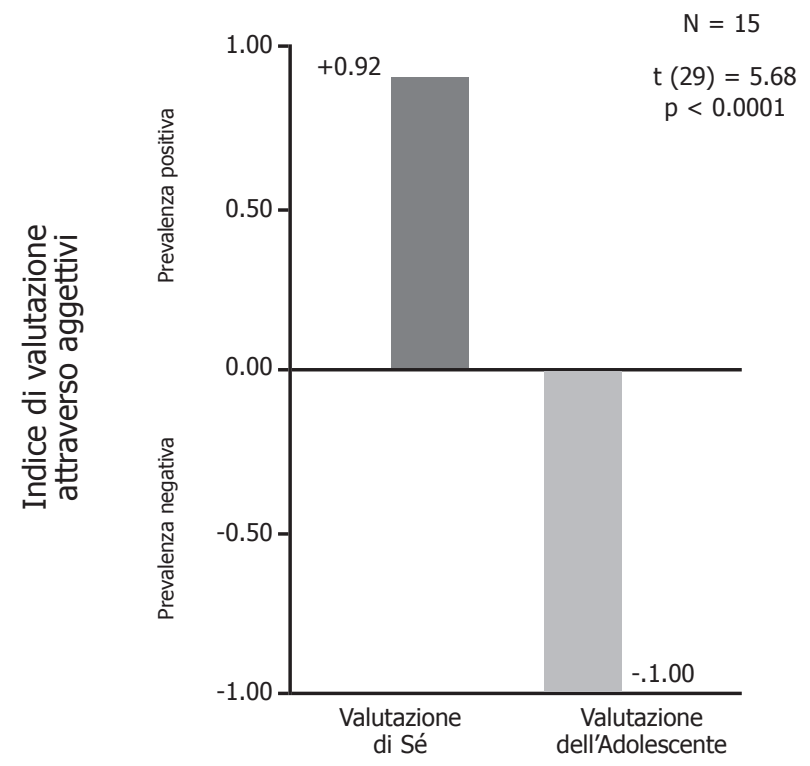

Figura 3. - Insegnanti che utilizzano elementi difensivi di tipo svalutante. 


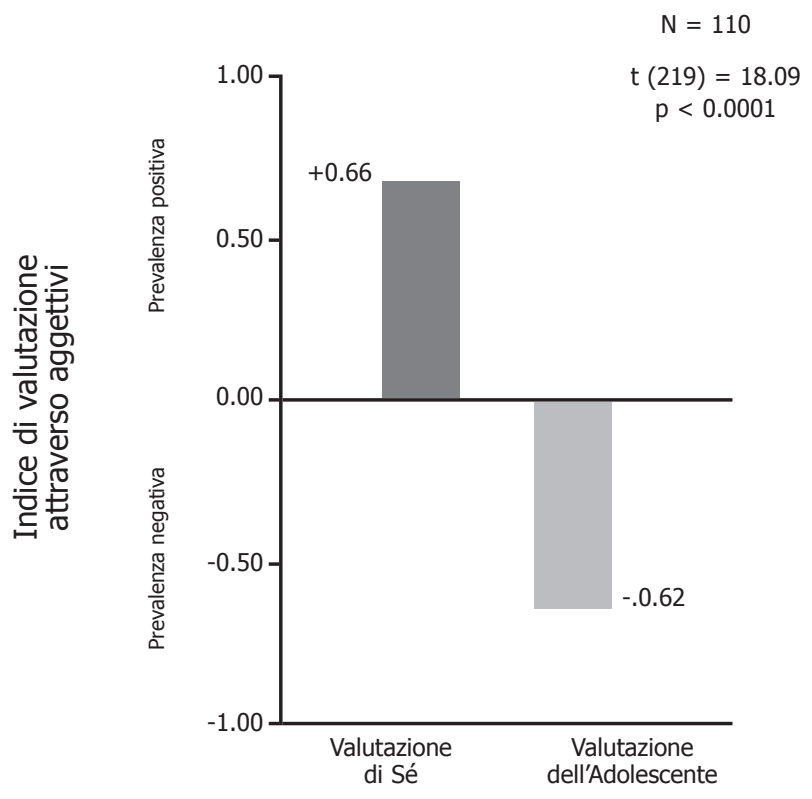

Figura 4. - Insegnanti con atteggiamento di tipo intermedio.

Naturalmente sarà utile, nell'ambito di uno sviluppo futuro di questo genere di indagini, verificare l'adozione dei meccanismi di difesa indicati con reattivi indipendenti, capaci di rilevarne la presenza: ciò permetterebbe di allargare l'indagine ad aspetti più profondi della personalità del docente, cui correlare la scelta di particolari strategie difensive, le quali, d'altra parte, come sappiamo, risultano adattive anche in funzione del contesto.

Le riflessioni conseguenti convergono, a questo punto, sul ruolo degli atteggiamenti dell'insegnante $\mathrm{e}$, in modo particolare, sul ruolo dei livelli individuali di tolleranza del conflitto (Bonaiuto, Giannini, \& Bonaiuto, 1990; Bonaiuto, Giannini, Biasi, \& Bartoli, 1996; Bartoli \& Bonaiuto, 1997); che possono rendere più o meno disponibili gli adulti ad accettare emotivamente le «bizzarrie» di certe condotte adolescenziali.

In altri termini, insegnanti più tolleranti (in genere meno ansiosi, meno rigidi, più flessibili e, in genere, anche maggiormente creativi) dovrebbero avvertire livelli minori di allarme e di emotività negativa nella relazione con gli adolescenti e quindi dovrebbero mostrare minori atteggiamenti difensivi e maggiori capacità di gestire in modo costruttivo la relazione educativa con allievi di questa fascia d'età. 
Da qui la necessità di una formazione del docente basata anche sullo sviluppo di competenze relazionali, accanto quelle più classiche di tipo didattico-valutativo, per la promozione di atteggiamenti funzionali alla comprensione empatica, basati sul rinforzo dell'intelligenza emotiva, delle conoscenze psicologiche in tema di evoluzione della personalità dell'allievo, e delle dinamiche psicologiche, anche talvolta difensive, che possono interessare la relazione educativa.

\section{LA FENOMENOLOGIA SPERIMENTALE QUALE SCIENZA DELLA SOGGETTIVITÀ BASATA SULLA VALIDAZIONE EMPIRICA: DALL'APPROCCIO CLASSICO AL NUOVO ORIENTAMENTO PSICODINAMICO INTEGRATO}

L'indagine appena presentata si inserisce nell'ottica della verifica quantitativa di assunti di matrice psicodinamica a spiccato valore soggettivo e qualitativo. Può costituire quindi una dimostrazione del valore dell'analisi fenomenologica quale scienza della soggettività, accompagnata in questo caso dall'interpretazione psicodinamica - quale ulteriore sviluppo dell'analisi fenomenica - e da una validazione empirica che ne sostiene il rilievo scientifico.

Questa analisi si pone sostanzialmente in linea con il postulato principe della cosiddetta Fenomenologia sperimentale che fu tra le prime correnti a pretendere la compresenza dei due canali di indagine: l'ermeneutica accompagnata dalla verifica empirica. Vengono, come abbiamo visto, proposti anche sostanziali sviluppi del paradigma funzionalista - con l'introduzione del livello di realtà univoca rispetto a quella ambigua -, integrazioni - secondo l'orientamento psicodinamico che supera il livello descrittivo della fenomenologia e si muove su quello interpretativo - e applicazioni in contesti diversi come quello educativo.

Per meglio comprendere i vantaggi che questa scelta teorico-metodologica rappresenta in termini di realismo, sia nel senso di una ricerca puntuale del riscontro empirico sia nell'ottica di un maggior controllo del grado di soggettività, è forse opportuno riassumere a grandi linee il cammino che ha caratterizzato nell'ultimo secolo la crescita dei saperi psicologici.

Storicamente con l'avvento della cosiddetta Psicologia della Gestalt o Psicologia della Forma, che traduce il tedesco Gestaltpsychologie, grazie al lavoro di eminenti studiosi come Wolfgang Köhler, Kurt Koffka, Max Wertheimer, Karl Dunker, Kurt Lewin, Wolfgang Metzger, Rudolf Arnheim, Cesare Musatti, Gaetano Kanizsa con i suoi allievi si sviluppò un orientamento che postulava l'indagine della totalità della vita psichica, l'importanza del contesto, la cosid- 
detta tendenza alla «buona forma». L'oggetto di studio riguardava le esperienze interiori ed il comportamento globale e veniva utilizzata una importante combinazione di metodi: l'analisi fenomenologica e la ricerca sperimentale. Ci si rivolgeva sia a soggetti non addestrati, sia a soggetti esperti e si indagavano in particolare configurazioni e qualità fenomeniche nella percezione, nell'immaginazione, nel movimento. Il postulato teorico assumeva la vita psichica come totalità, articolata su basi prevalentemente innate. La conoscenza risultava essere un processo attivo di organizzazione (concetto di "campo») e le configurazioni percettive si ritenevano effetto di «fattori formali»

Seguendo la successione storica, si è affermato negli Stati Uniti negli anni '40, il cosiddetto Funzionalismo, in aperta contrapposizione all'orientamento gestaltista, forse per sanare alcune carenze e forse anche per fare emergere e delineare la propria identità come movimento scientifico autonomo, studiando ciò che fino ad allora era stato in qualche modo trascurato.

Il Funzionalismo, che riprendeva alcune premesse del pensiero di Darwin e di Dewey, ma che può annoverare fra i precursori anche William James, Granville Stanley Hall, James McKean Cattell e, per certi aspetti, Hermann von Helmholtz, considerava prioritaria la funzionalità dei processi psichici per assicurare l'adattamento all'ambiente e quindi la sopravvivenza umana.

Postulava la vita psichica come basata su desideri, esigenze, bisogni che regolano anche i processi cognitivi e guidano i comportamenti. Tra i più noti rappresentanti vi sono stati eminenti studiosi come Jerome Bruner, Leo Postman, Elliott McGinnies, Gardner Murphy, Richard S. Lazarus, vari altri e, per certi versi, Egon Brunswik, fautore del cosiddetto Funzionalismo probabilistico, insieme alla moglie, Else Frenkel-Brunswik, studiosa dell'intolleranza dell'ambiguità e della rigidità percettiva e comportamentale.

L'oggetto di studio prioritario sono stati quindi i processi affettivi e cognitivi consapevoli e inconsapevoli. Veniva adottato il metodo sperimentale applicato su singoli soggetti e gruppi. L'ambito di ricerca riguardava in particolare i bisogni, le emozioni, le esperienze passate ed i loro riverberi sui processi cognitivi, anche in termini di difese percettive. L'ipotesi di partenza consisteva nel fatto che i bisogni influenzano per consonanza (assimilazione e proiezione) i processi cognitivi; ma, in base a quanto sappiamo oggi, va precisato che ciò avviene solo in condizioni di «ambiguità» della situazione da valutare. La «proiezione» dei bisogni avviene quindi solo in queste circostanze.

Grazie poi alla successiva ricerca in ambito fenomenologico-sperimentale e psicodinamico, è stato possibile oggi individuare alcuni limiti del funzionalismo originario e muovere alcune critiche costruttive che qui ci limitiamo a sintetizzare.

Motivazione e cognizione erano state collegate in modo unilaterale, ipotizzando soltanto l'assimilazione in condizioni di ambiguità. Non si tenne 
conto dei processi di contrasto, con enfatizzazione delle differenze rispetto alle aspettative, quando la situazione da valutare si presenta come univoca (Bonaiuto, Giannini, Biasi, \& Bonaiuto, 1995; Giannini, Biasi \& Bonaiuto, 1995; Bonaiuto, Giannini, \& Biasi, 2002). Trascurando quanto accade nelle condizioni di univocità percettiva, il funzionalismo classico non ha potuto spiegare che cosa accade quando i desideri si contrappongono alla realtà; e non ha tenuto conto del fatto che la motivazione alla conoscenza implica anche l'esigenza di realismo.

Rispondendo probabilmente ad una forte esigenza di scientificità, al fine forse di fugare angosce per la misurazione oggettiva rispetto a variabili troppo «soggettive», ed in linea anche con i valori socio-culturali dei vari periodi storici di riferimento, si ebbe poi l'epoca dell'avvento del Comportamentismo. Ricordiamo che questa corrente si è concentrata sullo studio del comportamento manifesto. Dal punto di vista metodologico si procedeva secondo l'osservazione diretta e la registrazione di atti comportamentali. Tra i maggiori studiosi vi sono stati l'americano Edward L. Thorndike, il russo Ivan P. Pavlov, e ancora, John B. Watson, Clark L. Hull, Burrus F. Skinner, Edward C. Tolman, e altri ancora. Al giorno d'oggi è facile muovere alcune obiezioni ai lineamenti professati da questo approccio teorico. Vi è la completa esclusione dello studio della coscienza accessibile tramite l'introspezione, non sono stati presi in considerazione i processi intra-personali. Vi è la mancata considerazione del ruolo delle rappresentazioni nel determinare il comportamento (tranne che nella versione del "comportamentismo intenzionale» di Tolman); e vi è la sottovalutazione del riconoscimento delle variabili affettive, le quali, anche quando sono prese parzialmente in considerazione (i cosiddetti drives), non sono studiate approfonditamente per la difficoltà di misurazione e la considerata aleatorietà delle differenze individuali.

Frutto del Comportamentismo e volto a superarne alcuni limiti epistemologici è sorto il Cognitivismo, attraverso il quale si è inteso studiare i processi mentali, non come semplici associazioni stimolo-risposta ma come complessi processi di elaborazione dell'informazione (Human Information Processing - HIP), fino a sviluppare l'interesse per le relazioni con le neuroscienze. Tra i maggiori rappresentanti vi sono stati Pewter H. Lindsay, Donald A. Norman, James J. Gibson, Ulrich Neisser e molti altri; fino a che negli ultimi trent'anni in Italia è stato difficile trovare qualche ricercatore che abbia escluso di essere in qualche modo un cognitivista.

L'oggetto di studio si è concentrato sui processi cognitivi consci e inconsci. Il metodo utilizzato ha riguardato la misurazione obiettiva delle prestazioni e la simulazione cibernetica. Venivano intervistati in genere campioni di soggetti statisticamente significativi. Gli ambiti di ricerca riguardavano soprattutto l'attenzione selettiva, la memoria, le immagini mentali. Si assu- 
meva che la mente umana fosse simile o equivalente al calcolatore, e appariva opportuno studiarne le caratteristiche seguendo questa analogia.

L'input allo studio dei processi cognitivi è stato storicamente di notevole rilievo grazie a questo approccio teorico. Vi sono tuttavia consistenti obiezioni e critiche costruttive da muovere anche a questo approccio, sia nella sua versione originale sia in quelle allargate.

Prima di tutto vi è stata una costante sottovalutazione dei processi affettivi (e quindi del ruolo della personalità) nell'elaborazione cognitiva, che va dall'intervento degli schemi mentali e delle aspettative allo studio dei meccanismi di difesa, il che ha portato a non considerare appieno la varietà e complessità della vita psichica.

Dal canto suo e con alterne vicende la Psicoanalisi, che si è affermata a latere di queste scuole di pensiero già enucleate, si è proposta come: (a) Metodo d'indagine; (b) Teoria di personalità; (c) Metodo terapeutico. Eminenti rappresentanti sono stati il fondatore, Sigmund Freud, inoltre Melanie Klein, Wilfried R. Bion, Erich Fromm, Sandor Ferenczi, Donald W. Winnicott, Bruno Bettelheim e ancora Carl Gustav Jung, Alfred Adler; o, in Italia, Cesare Musatti, Franco Fornari, Eugenio Gaddini, Francesco Corrao, Glauco Carloni e numerosi altri.

Come oggetto di studio la psicoanalisi ha considerato prioritarie le dinamiche mentali inconsce (relazioni con sintomi nevrotici e con il funzionamento psichico normale). Ha sviluppato un metodo originale basato appunto sull'introspezione psicoanalitica (in particolare con l'innovazione delle «associazioni libere»), su esperienze e dinamiche del profondo; sull'osservazione della condotta e/o di produzioni concrete, con interpretazioni in chiave psicodinamica. Gli ambiti di ricerca hanno riguardato i sogni, alcuni fenomeni della memoria (oblio, amnesie, atti mancati), il pensiero, le motivazioni profonde, le fasi dello sviluppo, le sindromi cliniche. Le ipotesi da cui si parte sono il determinismo psichico; l'influenza sul comportamento manifesto di fattori inconsci: istinti, conflitti, esperienze precoci, anche rimosse e dimenticate.

Sono state mosse varie critiche, talvolta feroci e distruttive, a tale approccio, fino ad avanzare dubbi e discussioni sulla sua "scientificità», sulla accessibilità dell'inconscio; accompagnate da accuse di «riduzionismo» per focalizzazioni settoriali nei processi di attribuzione causale («Edipo», "pulsione», ecc.) e da dubbi e discussioni sull'efficacia terapeutica (Hunt, 2007).

D'altra parte questi possono essere i rischi che gli studiosi di matrice psicoanalitica hanno in qualche modo accettato di correre al fine di tentare di spiegare (peculiarità e rischio dell'approccio psicodinamico) e non solo di descrivere (peculiarità e limite dell'approccio fenomenologico) fenomeni psichici anche complessi. 
Nel frattempo si era anche consolidata la posizione della cosiddetta $\mathrm{Fe}$ nomenologia sperimentale.

Un precursore è stato riconosciuto in Carl Stumpf (1873 e 1883). Vi hanno aderito alcuni studiosi italiani insieme ad altri americani e giapponesi. Fra i promotori abbiamo avuto in Italia Gaetano Kanizsa (1984), Paolo Bozzi (1989), insieme a colleghi ed allievi, quali Giovanni Vicario, Mario Zanforlin, Walter Gerbino, Manfredo Massironi, Marco Sambin, Osvaldo da Pos, ecc.

Il metodo è centrato sull'analisi fenomenologica accompagnata dalla sperimentazione. Le ipotesi allo studio vedono i processi psichici come determinati da variabili colte attraverso l'esame di vissuti autentici, rifiutando commistioni con gli approcci neurofisiologici o d'altro genere. Come ha riferito a suo tempo Bozzi, che ha sovente voluto esprimersi in latino, la percezione deve essere spiegata juxta propria principia (Bozzi, 1989 e 1993).

Le ricerche sono condotte nell'ambito della percezione, del pensiero, nonché delle emozioni e delle motivazioni anche attraverso apporti del nostro gruppo di ricerca (Bonaiuto, 1966; Bonaiuto, Biasi, \& Giannini, 2002) che ha così proposto una integrazione dell'analisi fenomenologica, della verifica sperimentale e della spiegazione psicodinamica ci viene da un orientamento più recente che qui infine presentiamo, con il termine di Approccio psicodinamico integrato.

Quest'ultimo approccio considera processi cognitivi e processi affettivi in stretta interdipendenza, e studia le leggi che ne regolano la dinamica, le caratteristiche dei fenomeni risultanti, le reciproche influenze. Postula la vita psichica e le relazioni tra fenomeni come descrivibili con linguaggio omogeneo. Considera importanti anche i fattori inconsapevoli nella determinazione dei processi psichici. L'approccio è limitato alla considerazione di variabili indipendenti esclusivamente di natura psichica. Rimane naturalmente aperto il problema delle relazioni con ambiti delimitati all'interno di altre discipline (fisiologia, sociologia, economia, ecc.).

L'oggetto di studio riguarda i processi psichici consapevoli e non consapevoli, i comportamenti umani normali nonché quelli eccezionali o abnormi.

Il metodo utilizzato implica come già detto, l'analisi fenomenologica, la riflessione psicodinamica le procedure sperimentali. Vi è particolare attenzione per l'allestimento di scale di valutazione e di strumentazioni appropriate: il che richiede notevole impegno innovativo. Vengono utilizzati campioni di singoli soggetti e gruppi. L'ambito di ricerca comprende i fenomeni percettivi, cognitivi, affettivi, motori e lo studio delle loro dinamiche, in relazione anche ai tratti di personalità. Le ipotesi allo studio considerano i processi psichici come influenzati da fattori sia personali, sia configurazionali. Le motivazioni vengono attivate o disattivate in funzione di informazioni ricevute e di prestazioni svolte. Motivazioni, atmosfere emotive e schemi mentali influi- 
scono sui fenomeni percettivi con modalità opposte a seconda delle condizioni di osservazione (ambigue o univoche). Le emozioni «negative» e gli stati di sofferenza, fastidio, eccessivo conflitto, mobilitano meccanismi difensivi, fra cui l'attribuzione di causalità e responsabilità, la ripulsa o la sottovalutazione in ambito estetico, ecc.

Numerosi studi sono stati condotti secondo queste linee negli ultimi trent'anni dal nostro gruppo di ricerca, in particolare con la guida di Paolo Bonaiuto. Possiamo considerare tale orientamento teorico uno sviluppo storico e tematico degli iniziali contributi forniti dalla scuola gestaltista (raccolti in Italia in particolare da Gaetano Kanizsa), debitamente integrati e rivisti; del movimento psicoanalitico diffuso in Italia da Cesare Musatti, che ha potenziato lo studio dei processi psichici secondo la visione dinamica, nonché della prospettiva integrata tra psicologia generale e clinica (secondo cui lo studio dei processi psichici prevede un continuum tra normalità a patologia), rappresentata negli anni '60, in Italia, da Renzo Canestrari.

Tale paradigma è risultato particolarmente fecondo per lo studio dei processi affettivi, argomento che è stato storicamente, come abbiamo visto, soggetto a riduzionismi da parte delle scuole cognitiviste e comportamentiste, caratterizzate da relativa chiusura e forti titubanze riguardo alle possibilità di misurare e manipolare in sede sperimentale variabili fluide quali le motivazioni e le emozioni. Accanto al rifiuto di studiare sistematicamente il mondo delle esigenze ed alla proclamazione della scelta teorica e metodologica di considerare come scientifica solo la rilevazione oggettiva del "comportamento manifesto", tuttavia già Pavlov nel produrre il cosiddetto "condizionamento classico» faceva leva sul bisogno di cibo dell'animale, sulle sue esigenze nutritive e quindi su dinamiche istintuali, altresì etichettabili come istanze pulsionali.

Si deve storicamente a Freud (1900) l'affermazione che «tutto il comportamento è motivato»; e l'individuazione di varie strategie metodologiche per analizzare gli scopi consapevoli o inconsapevoli che orientano atti, desideri, fantasie (analisi dei sogni, degli atti mancati, dei lapsus, ecc.). Dopo anni di "oscurantismo», durante i quali le scoperte freudiane dell'inconscio e delle dinamiche affettive restarono, per così dire, ai margini dell'indagine scientifica, la moderna psicologia ha tenuto conto delle variabili affettive nell'ambito delle elaborazioni teoretiche e vari autori hanno proceduto a verifiche sperimentali dell'intervento di tali fattori nel comportamento umano (Lewin, 1935; Ancona, 1958; Berlyne, 1960; Nuttin, 1965; Bonaiuto, 1967, 1970; Heckhausen, 1991; Bartoli \& Bonaiuto, 1997; Pawlik \& d'Ydewalle, 2006; Bonaiuto, Biasi, \& Giannini, 2002; Biasi, 2010 e molti altri).

Come ormai dimostrato da un'ampia casistica sperimentale raccolta negli ultimi vent'anni da questo gruppo di ricerca romano, tali esigenze parteci- 
pano alla elaborazione cognitiva delle informazioni e generano, in base al grado di conflittualità individuale, processi di difesa elo di facilitazione. Processi affettivi e cognitivi si collegano secondo un continuum che va dalla normalità alla patologia e che connette emozioni, motivazioni, interessi, atteggiamenti, tratti di personalità. Tale orientamento teorico integrato vede propria una scelta metodologica multipla che, come già riferito, è basata sull'analisi fenomenologica, l'interpretazione psicodinamica e la verifica empirica, nell'intento non solo rilevare ma, in particolare, misurare il "portato della soggettività», rientrando così a pieno titolo, riteniamo, nell'ottica del nuovo realismo.

A questo proposito appare possibile a nostro avviso tracciare un parallelo tra approcci teorici, oggetti di studio e metodi prescelti in ambito scientifico. Vediamo cioè che a seconda dell'orientamento teorico seguito ogni studioso seleziona alcuni argomenti di studio ed adotta metodologie congruenti finendo inevitabilmente per trascurare altre metodologie meno adatte ad affrontare quel particolare oggetto di studio, soprattutto se non in linea con i postulati iniziali.

I dati ottenuti grazie alle varie analisi condotte vengono via via inseriti in schemi interpretativi che dipendono anche dalla formazione acquisita dallo studioso. In particolare la conoscenza delle dinamiche affettive - in funzione della dotazione istintuale, dell'età, dell'educazione, della cultura, in altri termini della personalità - opera sia nel soggetto osservato, sia nello stesso osservatore.

Si può affrontare a nostro avviso tale effetto complesso appellandosi al metodo sperimentale quando possibile o, comunque, a metodologie empiriche, ed anche alla convergenza di più metodologie (qualitative e quantitative), che, partendo da paradigmi teorici diversi, esplorano l'oggetto di studio da vari punti di vista, ciò a garanzia di una maggiore generalizzabilità dei risultati, per il raggiungimento di una maggiore validità scientifica.

\section{RIFERIMENTI BIBLIOGRAFICI}

Amann Gainotti, M. (2002). Lo sviluppo cognitivo durante l'adolescenza. Roma: Seam. Ancona, L. (1958). La motivation au point de vue clinique. Contrib. Ist. Psicol. Univ. Catt. Milano, 23.

Balcetis, E., \& Dunning, D. (2006). See what you want to see: Motivational influences on visual perception. Journal of Personality and Social Psychology, 91(4), 612-625.

Bartoli, G., \& Bonaiuto, P. (1997). Psicodinamica e sperimentazione. Roma: N.I.S. Carocci. 
Berlyne, D. E. (1960). Conflict arousal and curiosity. New York: McGraw-Hill.

Biasi, V. (2006). Il conflitto psichico. Analisi fenomenologiche e verifiche sperimentali. Roma: Monolite.

Biasi, V. (2010). Processi affettivi e dinamiche della conoscenza. Milano: Guerini e Associati.

Biasi, V., Bartoli, G., \& Amann Gainotti, M. (2004). Rappresentazioni e aspettative degli insegnanti in tema di adolescenti. Relazioni tra autovalutazione, valutazione dell'altro e difese psichiche. In M. Amann Gainotti \& V. Biasi (a cura di), Essere insegnanti in classi di adolescenti (pp. 209-222). Milano: Guerini e Associati.

Biasi, V., Bartoli, G., \& Squarcia, R. (2000). Lineamenti di personalità, stili educativi e identità degli insegnanti. Cadmo, 23(8), 17-39.

Biasi, V., Calcagni, S., \& Parsi, M. (2008). Conflict overloading and perceptual defence in experiments with architectural depictions. In K. S. Bordens (Ed.), Symposium IV: The use of architectural models for studying visual perception, Proceedings of the 20th Biennial Congress of the International Association of Empirical Aesthetics (pp. 314-316). Chicago, IL: IAEA.

Biasi, V., Chiappetta Cajola, L., \& Bonaiuto, P. (2010). Valutare la formazione degli schemi mentali nei disturbi dell'apprendimento / Evaluating the formation of mental schemata in learning disorders. Journal of Educational, Cultural and Psychological Studies, 2, 117-138.

Bonaiuto, P. (1965). Tavola d'inquadramento e di previsione degli «effetti di campo» e dinamica delle qualità fenomeniche. Giornale di Psichiatria e Neuropatologia, 42(4), Suppl., 1443-1685.

Bonaiuto, P. (1966). Lineamenti d'indagine fenomenologica sperimentale in rapporto con problemi ed esperienze della progettazione visuale. Il Verri, 22, 24-65.

Bonaiuto, P. (1967). Le motivazioni dell'attività nell'età evolutiva. Analisi fenomenologica, riferimenti e indicazioni per la sperimentazione. In AA.VV., La problematica dei campi da gioco nell'età evolutiva. Impostazione di proposte di progettazione, $2^{\circ}$ Concorso di Idee del Centro Milanese per lo Sport e la Ricreazione (C.M.S.R.), Milano. Pubbl. anche a cura del C.I.G.I., Ivrea, 1970 (ristampa, Kappa: Roma).

Bonaiuto, P. (1970). Sulle ricerche psicologiche europee in tema di monotonia percettiva e motoria («Sensory deprivation» e simili). Il processo della saturazione di qualità fenomeniche. Rassegna di Neuropsichiatria, 24(3-4), 1-114.

Bonaiuto, P. (1984). Equivalenze spazio-tempo nel sovraccarico percettivo. Comunicazioni scientifiche di psicologia generale. Scritti in onore di Ernesto Valentini, 12, 371-402.

Bonaiuto, P., \& Bartoli, G. (1967). Ricerca su esigenze manifeste in preadolescenti di ambiente urbano. Molteplicità degli interessi e tendenze all'integrazione 
degli ambiti d'attività in luogo di dicotomie rigide. Rassegna di Psicologia Generale e Clinica, 8, 3-56.

Bonaiuto, P., \& Bartoli, G. (1970). Caratterizzazione del fanciullo, del preadolescente e dell'adolescente, mediante indagini sugli interessi, in differenti socioculture. Minerva Pediatrica, 22(44), 2156-2163.

Bonaiuto, P., Biasi, V., \& Giannini, A. M. (2002). Fenomenologia sperimentale e motivazioni umane. Teorie \& Modelli, 7(2-3), 267-288.

Bonaiuto, P., Biasi, V., Giannini, A. M., Calcagni, S., \& Bellini, D. (2007). Emotional influences on the perception of architectural incongruities. Communication presented at Giornata di studio sulle Emozioni, Padova. Abstract in V. L. Zammuner \& C. Galli (a cura di), Giornata di studio sulle Emozioni 2007, Padova. Proceedings. Padova: CLEUP (CD-Rom).

Bonaiuto, P., Biasi, V., Giannini, A. M., Calcagni, S., \& Parsi, M. (2008). Conflict overload effect manipulation and the perception of architectural incongruities. Communication presented at the IAPS Conference, Roma.

Bonaiuto, P., Giannini, A. M., Biasi, V., \& Bartoli, G. (1996). Stili cognitivi e atteggiamenti verso la trasgressione di regole sportive. In G. V. Caprara \& G. P. Lombardo (a cura di), Temi di psicologia dello sport (pp. 57-93). Roma: C.O.N.I. - Università degli Studi di Roma «La Sapienza».

Bonaiuto, P., Giannini, A. M., Biasi, V., \& Bonaiuto, M. (1995). Emphatization and normalization phenomena in social communication of incongruous colours. In C. J. Hawkyard (Ed.), Colour communication (pp. 218-221). Manchester: U.M.I.S.T.

Bonaiuto, P., Giannini, A. M., Biasi, V., Miceu Romano, M., \& Bonaiuto, M. (1997). Visual defense or facilitation processes favored by alarming or playful colours. In C. M. Dickinson, J. Murray, \& D. Carden (Eds.), John Dalton's colour vision legacy (pp. 723-731). London: Taylor \& Francis.

Bonaiuto, P., Giannini, A. M., \& Bonaiuto, M. (1990). Piloting mental schemata on building images. In A. Fusco, F. Battisti, \& R. Tomassoni (Eds.), Recent experiences in general and social psychology in Italy and Poland (pp. 85-129). Milano: Franco Angeli.

Bozzi, P. (1989). Fenomenologia sperimentale. Bologna: Il Mulino.

Bruner, J. S. (1992). Another look at new look 1. American Psycologyst, 47(6), 740743.

Bruner, J. S., \& Goodman, C. C. (1947). Values and needs as organizing factors in perception. Journal of Abnormal Social Psychology, 42, 33-44.

Bruner, J. S., \& Postman, L. (1947). Emotional selectivity in perception and reaction. Journal of Personality, 16, 69-77.

Bruner, J. S., \& Postman, L. (1949a). On the perception of incongruity: A paradigm. Journal of Personality, 18, 206-223. 
Bruner, J. S., \& Postman, L. (1949b). Perception, cognition and behaviour. Journal of Personality, 18, 14-31.

Canestrari, R. (1955). Il funzionalismo nella percezione. Rivista di Psicologia, 49, 65-95.

Canestrari, R. (1970). Osservazioni su conflitti psichici nell'adolescenza. Minerva Pediatrica, 22, 2091-2111.

Canestrari, R., \& Marzi, A. (1958). Motivazione e percezione nella psicologia della personalità. In F. Rossi-Landi (a cura di), Il pensiero americano contemporaneo. Scienze sociali (pp. 75-115). Milano: Edizioni di Comunità.

Cramer, P. (2000). Defense mechanisms in psychology today. American Psychologist, 55(6), 637-646.

Disanto, A. M. (2002). Una sfida. Insegnanti e adolescenti a confronto. Roma: Borla.

Dolto, F. (1996). Solitudine felice (trad. it., Milano: Mondadori).

Erdelyi, M. H. (1974). A new look at the new look: Perceptual defense and vigilance. Psychological Review, 81, 1-25.

Fonzi, A. (a cura di). (2002). Star male a scuola. Indicatori e correlati del disagio scolastico. Età Evolutiva, 71(1), 54-128.

Freud, S. (1900). Die Traumdeutung. Leipzig: Deuticke.

Giannini, A. M., Biasi, V., \& Bonaiuto, P. (1995). Un nuovo esperimento sui processi percettivi di attenuazione o risalto di anomalie strutturali, influenzati dal colore. Comunicazioni Scientifiche di Psicologia Generale, 13, 101-132.

Heckhausen H. (1964). Complexity in perception: Phenomenal criteria and information theoretic calculus. A note on D.E. Berlyne's "Complexity Effects». Canadian Journal of Psychology, 18, 168-173.

Holyoak, K. J., \& Morrison, R. G. (Eds.). (2005). The Cambridge handbook of thinking and reasoning. New York: Cambridge University Press.

Kanizsa, G. (a cura di). (1984). Fenomenologia sperimentale della visione. Milano: Franco Angeli.

Lenhart, L. A., \& Rabiner, D. L. (1995). An integrative approach to the study of social competence in adolescence. Development \& Psychopathology, 7(3), 543561.

Levine, R., Chein, I., \& Murphy, G. (1942). The relation of the intensity of a need to the amount of perceptual distortion: A preliminary report. The Journal of Psychology: Interdisciplinary and Applied, 13, 283-293.

Lewin, K. (1935). A dynamic theory of personality. New York: McGraw-Hill.

Lingiardi, V., \& Madeddu, F. (1994). I meccanismi di difesa. Teoria clinica e ricerca empirica. Milano: Cortina.

McClelland, D. C., \& Atkinson, J. W. (1949). The projective expression of needs. I. The effect of different intensities of the hunger drive on perception. Journal of Psychology, 27, 311-330. 
Molden, D. C., \& Higgins, E. T. (2005). Motivated thinking. In K. J. Holyoak \& R. G. Morrison (Eds.), The Cambridge handbooh of thinking and reasoning (pp. 295-317). New York: Cambridge University Press.

Nuttin, J. (1965). La motivation. Paris: PUF.

Palmonari, A. (a cura di). (2001). Adolescenza. Bologna: Il Mulino.

Parker, J. D. A., \& Wood, L. A. (2008). Personality and the coping process. In G. J. Boyle, G. Matthews, \& D. H. Saklofske (Eds.), The Sage handbook of personality theory and assessment (pp. 506-519). London: Sage.

Pawlik, K., \& D’Ydewalle, G. (2006). Psychological concepts. An international historical perspective. New York: Psychology Press - Taylor \& Francis.

Perry, J. C., \& Cooper, S. H. (1987). Empirical studies of psychological defenses. In R. Michels \& J. Cavenar (Eds.), Psychiatry, Vol. 1 (ch. 30). Philadelphia, PA: Lippincott.

Pietropolli Charmet, G. (2000). I nuovi adolescenti. Padri e madri di fronte a una sfida. Milano: Cortina.

Postman, L., Bruner, J. S., \& McGinnies, E. (1948). Personal values as selective factors in perception. Journal Abnormal, Social Psychology, 43, 142-154.

Schafer, R., \& Murphy, G. (1943). The role of autism in a visual figure-ground relationship. Journal of Experimental Psychology, 32, 335-343.

Theios, J., \& Jenison, R. (1999). A signal detection theory analysis of an unconscious perception effect. Perception \& Psychophysics, 61(5), 986-992.

Vaillant, G. E. (1992). Ego mechanisms of defense. Washington, DC: American Psychiatric Press.

Vanderplas, J. M., \& Blake, R. R. (1949). Selective sensitization in auditory perception. Journal of Personality, 18, 252-266.

Weinberger, J., Siegel, P., \& Decamello, A. (2000). On integrating psychoanalysis and cognitive science. Psychoanalysis \& Contemporary Thought, 23(2), 147-175.

Wispè, L. G., \& Dranbarean, N. C. (1953). Psychological need, word frequency and visual duration thresholds. Journal of Experimental Psychology, 46, 25-31.

\section{Riassunto}

Il presente contributo esamina, nell'ottica di un'integrazione tra Fenomenologia sperimentale e assunti psicodinamici, il passaggio dall'analisi introspettiva basata sull'applicazione del metodo fenomenologico, alla valutazione dei dati introspettivi raccolti attraverso il ricorso a metodi empirici che definiscono il terreno della cosiddetta «Fenomenologia sperimentale». Tale esame viene condotto in quest'occasione nell'area dei meccanismi di difesa psichica ove vengono delineati i processi contrapposti della "difesa percettiva" e della enfa- 
tizzazione (facilitazione, risalto). Dopo la descrizione dei principali risultati conseguiti in ambito percettologico, laddove è possibile permettersi il lusso di un protocollo sperimentale ortodosso, l'attenzione viene spostata su indagini condotte sul campo in ambito educativo per quanto concerne la relazione docente-allievo, secondo una metodologia empirica che permette di "quantificare" la presenza di specifici meccanismi di difesa che possono intervenire nella relazione educativa, quali la sovrastima o la sottostima delle qualità fenomeniche attribuite a sè elo all'altro. Oltre ad una conferma delle principali dinamiche di enfatizzazione (sovrastima) e difesa (sottostima) dimostrate in sede sperimentale attraverso indagini di laboratorio, si vuole offrire un esempio di quantificazione di un dato sostanzialmente qualitativo quale quello rappresentato dal concetto di "meccanismo di difesa", come appare già nella sua definizione: si definisce meccanismo di difesa qualunque strategia comportamentale o atteggiamento atto a ridurre elo prevenire il dolore e la sofferenza soggettivamente percepita. L'occasione è ghiotta dal punto di vista metodologico per dare dimostrazione di come sia possibile quantificare variabili a forte valenza soggettiva, al fine di ottenere una verifica empirica, momento indispensabile per il raggiungimento di conoscenze accreditabili sul piano scientifico.

Parole chiave: Fenomenologia sperimentale, Difesa psichica, Percezione, Psicodinamica, Relazione educativa. 